\title{
Effect of electromagnetic forces on aluminium cast structure
}

\author{
UDC: $669.715-14: 548.52: 538.69$
}

\section{T. A. El-Bassyouni*, A. A. Badr**, A. N. Abdel-Azim***, J. Lakner**** and Z. Olah****}

Molten commercial aluminium was oast in sand mould in the presence of high electric current. Grain refinement was enhanced as the current was increased. In case of alternating external magnetic field, however, there was no proportionality between the refinement and magnetic field strength. The ingot acquired fine equiaxed grains when the magnetic field was about 0.3 kilo gauss. At all other values, ingots showed mixed structure of equiaxed and columnar areas to varying proportions. The ingot cast in this experiment was about $100 \mathrm{~g}$.

The behaviour of the 4-kg ingot cast in an experimental semi-continuous casting machine was almost the same. The number of grains $/ \mathrm{cm}^{2}$ in both experiments was about 700 .

\section{Introduction}

The origin and variations in cast structure are explained by nucleation and growth phenomenon and heat flow effects and have been ably reviewd (1). In addition, some dynamic factors such as mechanical vibrations $(2,3)$, ultrasonics (4) and magnetic fields (5) have been shown to decrease grain size of the equiaxed zone and reduce the volume of the columnar zone.

The refinement due to electromagnetic forces is attributed to the electromagnetically induced motion through the melt. The latter arises from the interaction of the current with its self-induced magnetic field. It can as well be due to the interaction of external alternating magnetic field with the current resulting from its induced emf.

\subsection{Flow of current through the melt}

The flow of electric current through a melt creates an encircling magnetic field, and the magnetic field and the current interact to produce an inward acting force, as shown in Fig (1a). This force acts on the current oarrying fluid to force it inward and, thus, a pressure builds up in the fluid that becomes equal and opposite to the Lorentz force. The latter is proportional to the product of the current density, $j$, and the field strength, $H$ :

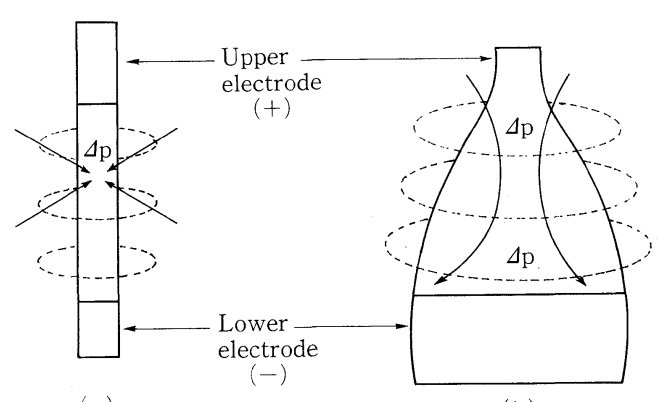

(a) (b)
Fig. 1 The increase of pressure in the fluid due to Lorentz force.

a) $P$ is the same at any level in the fluid.

b) $P$ at the top is larger than $P$ at the bottom.

*, *** Prof. and Researcher, CMRDI, Cairo.

** Chief of Material Technology Dept., MTC, Cairo.

**** Researchers at ALLUTERV-FKI, Hungary. 


$$
\Delta P \propto j \cdot H
$$

Since the field strength is proportional to the current, $I$,

$$
\Delta p \propto j \cdot I
$$

When the current density is decreased by increasing the cross-sectional area of the lower electrode, $\Delta p$ near the bottom will be smaller than $\Delta p$ at the top.

Thus, a pressure gradient and, hence, a flow of fluid occurs as shown in Fig (1b), If the flow is laminar, then the flow velocity is proportional to the pressure, so that:

$$
v \propto j \cdot I
$$

If the constricted area over which the current emerges is fixed, then:

$v \propto I^{2}$

This Lorentz-force-induced motion is independent of current polarity and occurs with AC as with DC. The results arrived at in this investigation are explainable on the basis of the foregoing discussion.

Electromagnetic casting introduced by the Russians ( 7 ) to commercial production uses medium frequency current passing through an inductor ring instead of letting the current to flow through the melt. This method results in an exceptionally smooth ingot surface since the molten metal has no contact with the mould wall.

\subsection{External magnetic field}

Electromagnetically induced motion arises from the interaction of the electric current with its selfinduced magnetic field. It can be intensified by applying an external magnetic field. Such a field can be used either to produce more intense motion at a given current, or to lower the current required to produce a given motion. This latter effect is useful where it is desired to reduce the resistance heating arising from the flow of current through the melt.

Direct magnetic field alone was found to suppress the motion caused by the natural convection in molten metal and thus enhances columnar growth $(6,8-10)$. The body force due to alternating magnetic field alone was found to be too small to cause moderate fluid motion. Thus, the macrostructure was not quite refined (8). The application of both direct magnetic and electric currents and of both alternative magnetic and electric currents were found to make the structure remarkably fine $(8,11)$. The values of the magnetic

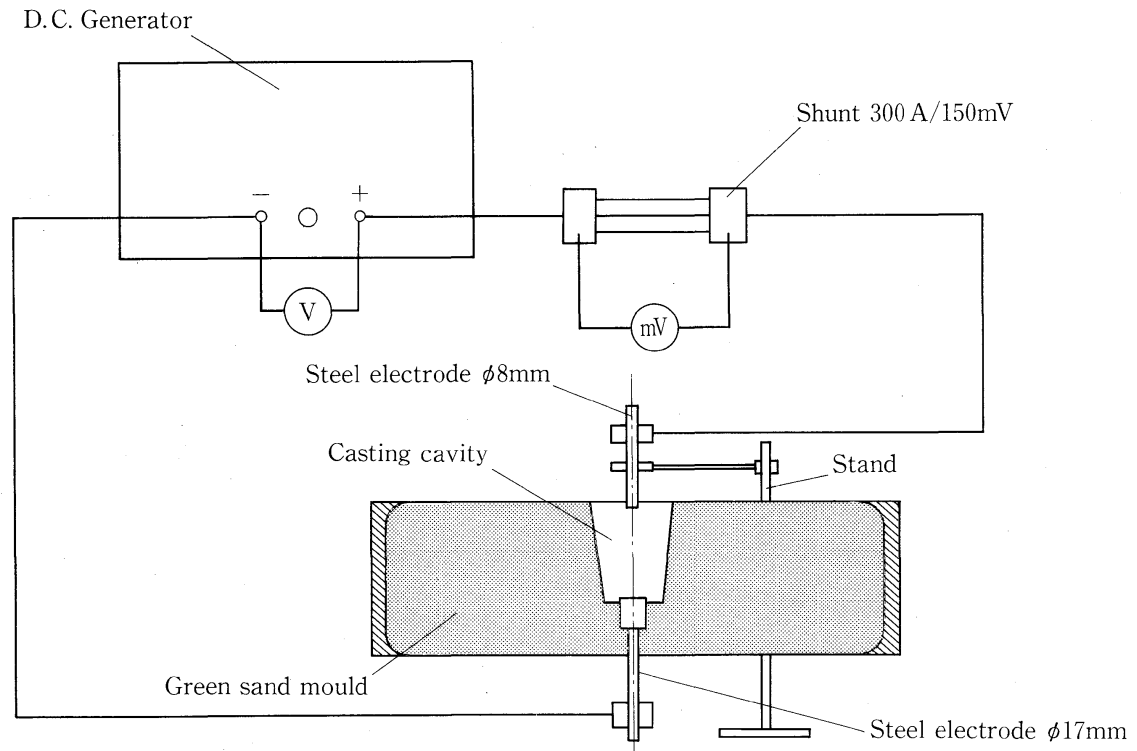

Fig. 2 The set up used for direct current experiment. 


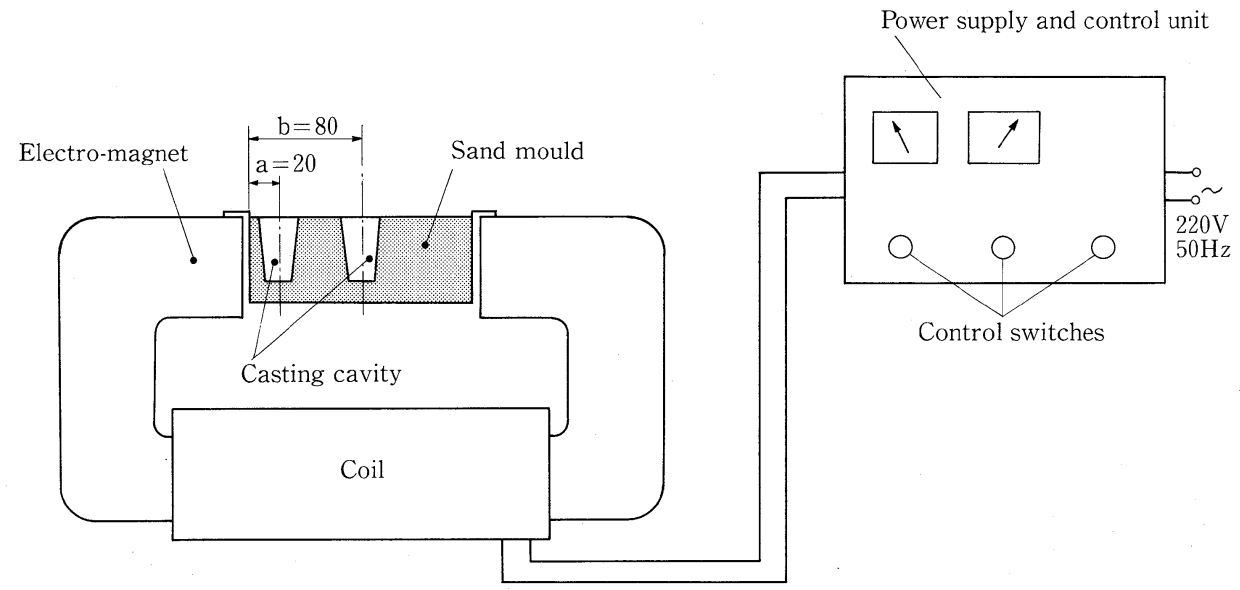

Fig. 3 The set up used for small ingots cast in alternating magnetic field.

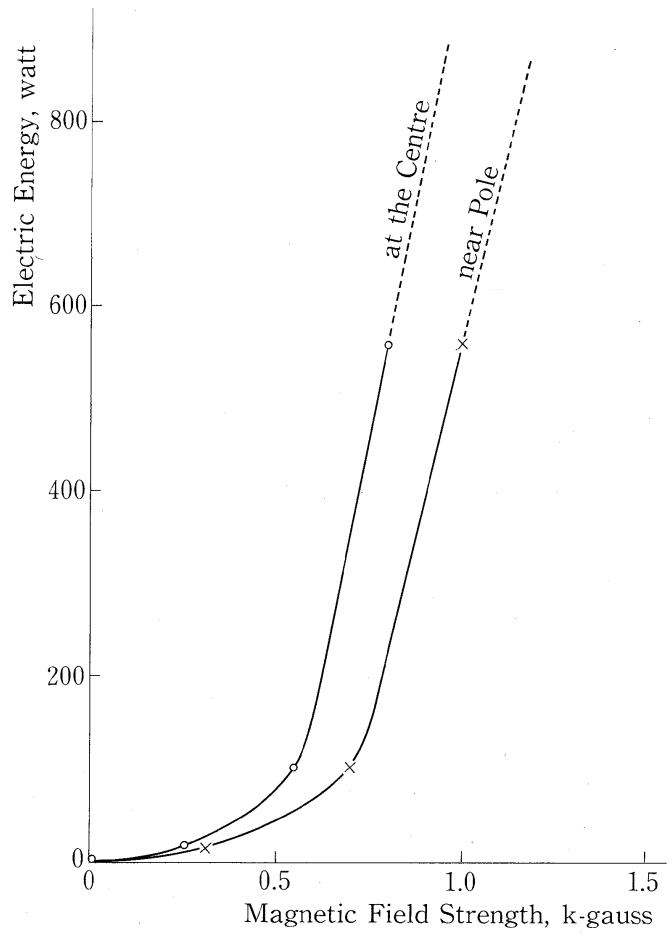

Fig. 4 Magnetic field intensity in case of small ingots measured at: a) $20 \mathrm{~mm}$ from the pole. b) midway between the two poles.

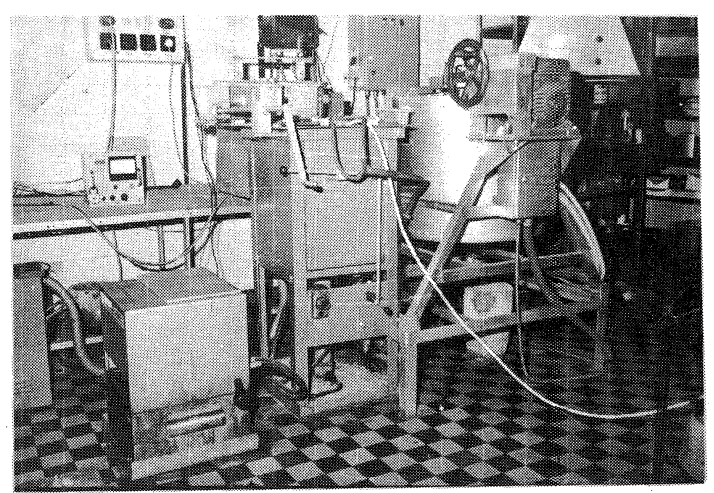

(a)

Inducter

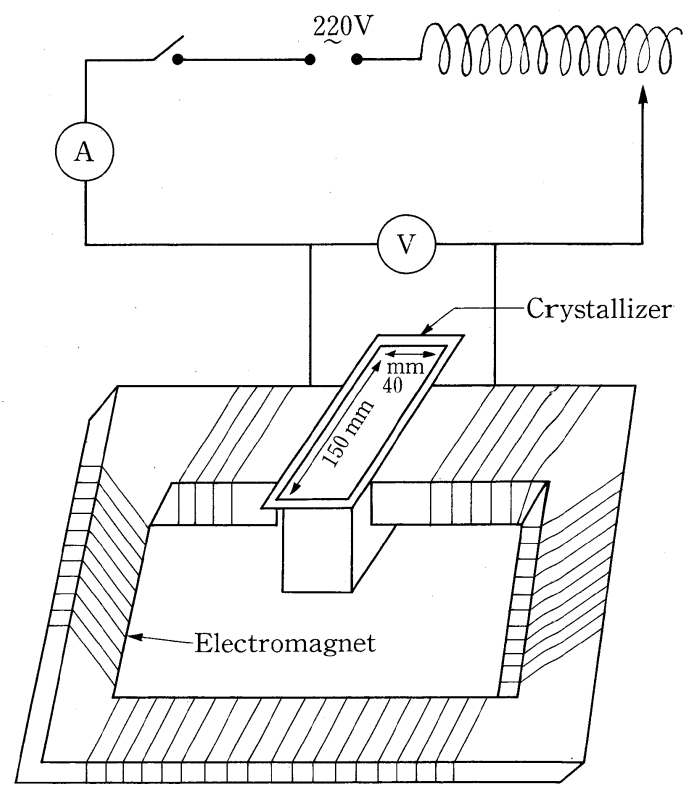

(b)

Fig. 5 The apparatus used to cast the 4- kg ingot: a) A photographic plate. b) Schematic drawing. 
field intensity reported in the literature were 4 (8), 5.4, 7.9 and 10.4 kilo gauss (12).

In this investigation, it was thought that alternating magnetic field alone can refine the structure contrary to the findings of Asai et al (8). The experimental results confirmed this thinking.
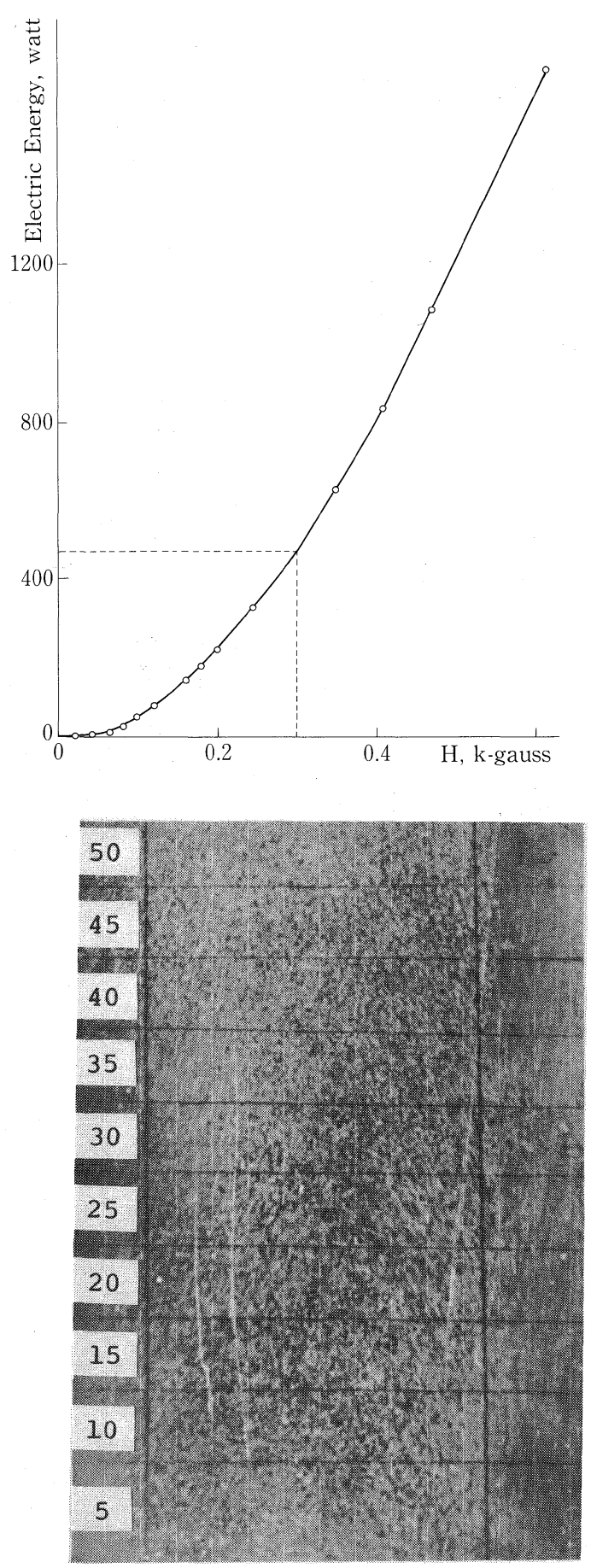

(a)
Fig. 6 Magnetic field intensity as a function of electric energy in case of large ingots.

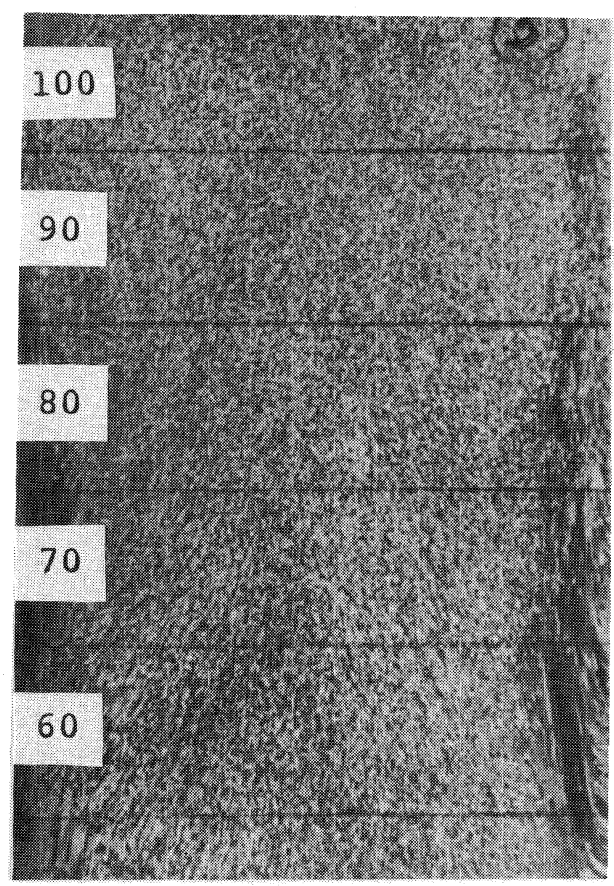

(b)

Fig. 7 Ingot cast under voltages varied from 5 ( $H=0.09 \mathrm{k}$-gauss $)$ to 60 volts $(0.27 \mathrm{k}$-gauss). Magnification $=0.6$

b) The voltage was changed from 50 (0.24 k-gauss) to 110 volts $(0.38 \mathrm{k}$-gauss). Magnification $=0.6$ 


\section{Experimental}

\subsection{Direct current}

The aluminium used contained $0.25 \% \mathrm{Fe}$ and $0.07 \% \mathrm{Si}$. Normal green sand moulding was used. The ingot was in the form of a tapered cylinder whose upper and lower diameters and height were 30, 25 and $50 \mathrm{~mm}$ respectively. The set up used for this experiment is shown in Fig (2). A DC welding generator was used to provide the high current. The diameter of the upper electrode was increased gradually until it did not show excessive heating during the passage of the current. The suitable diameter was found to be $8 \mathrm{~mm}$. The diameter of the lower electrode was $18 \mathrm{~mm}$.

\subsection{External alternating magnetic field}

In this experiment, casting was carried out in green sand mould in the presence of alternating magnetic field of $50 \mathrm{~Hz}$. The installation used in this case is presented in Fig (3). A preliminary experiment was carried out to measure the magnetic field intensity at position (a), $20 \mathrm{~mm}$ from the pole, and position (b), $80 \mathrm{~mm}$ from the pole and its dependence on the electric energy input. The results are shown in Fig (4). The aluminium was molten in a crucible furnace and the pouring temperature was kept at $720^{\circ} \mathrm{C}$. The value of the electric energy at which maximum refinement occurred was sought and the magnetic field intensity determined.

The 4-kg ingot was cast in a semi-continuous casting machine in the presence of a $50 \mathrm{~Hz}$ alternating magnetic field. The set up used is photographed in Fig (5a) and demonstrated schematically in Fig (5b).

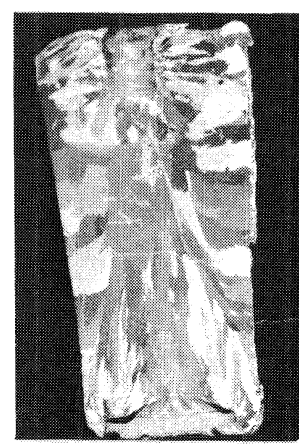

(a)

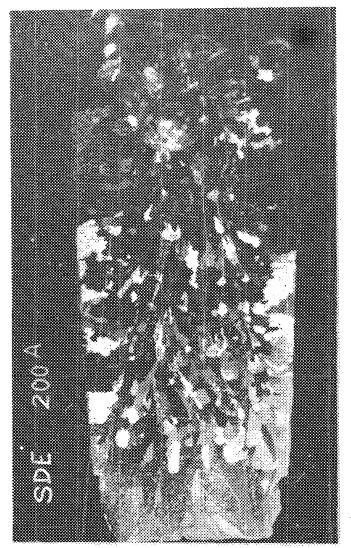

(d)

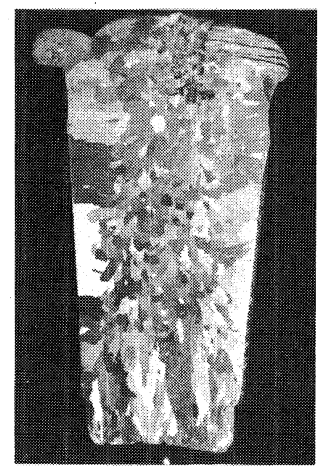

(b)

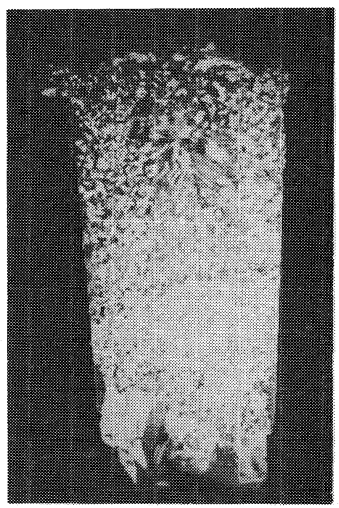

(e)

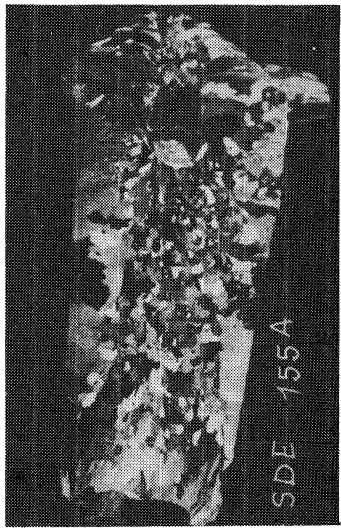

(c)

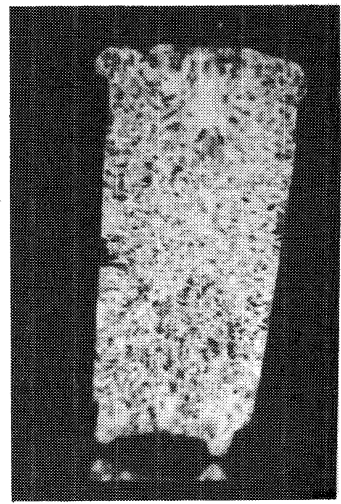

(f)

Fig. 8 Macrostructures of ingots subjected to the following direct currents: a) zero,b) 100,c) 155,d) 200,e) 250, f) 460 A.Magnification $=1$ 
The pouring temperature was $720^{\circ} \mathrm{C}$. The rate of cooling water was $27 \mathrm{l} / \mathrm{min}$. The rate of downward withdrawal of the ingot was $125 \mathrm{~mm} / \mathrm{min}$. The values of the voltage together with the corresponding values of the current were recorded. Knowing the current, the magnetic field strength could be calculated from the following formula:

$$
H=\frac{I \cdot N}{\left(\frac{\mu_{0}}{\mu}\right) l+\delta}
$$

where: $I=$ effective current

$$
\begin{aligned}
N & =\text { number of turns } \\
\mu_{0} & =\text { permeability in space } \\
\mu & =\text { permeability in aluminium } \\
l & =\text { length of the magnet core } \\
\delta & =\text { gap width. }
\end{aligned}
$$

The magnetic field intensity was calculated in terms of electric energy input and the relation is plotted in Fig (6).

A preliminary experiment was carried out to determine quickly the energy required to give the most refinement. In one ingot the voltage was changed during casting and the area of each effective voltage was marked on the ingot as shown in Fig (7a). Knowing the current corresponding to each voltage, the electric energy input was calculated and the corresponding magnetic field strength can be read directly from Fig (6). The value of the magnetic field strength in this case was in the range 0.09 to 0.27 kilo-gauss. Another ingot was cast and the magnetic field strength was changed from 0.24 to 0.38 kilo-gauss as shown in Fig ( 7 ). The maximum refinement appeared to be between 0.27 and 0.33 kilo-gauss. Three more ingots were cast while the magnetic field strength was kept constant at either $0.28,0.30$ or 0.33 kilo-gauss respectively. Another ingot was cast in the absence of magnetic field for comparison.

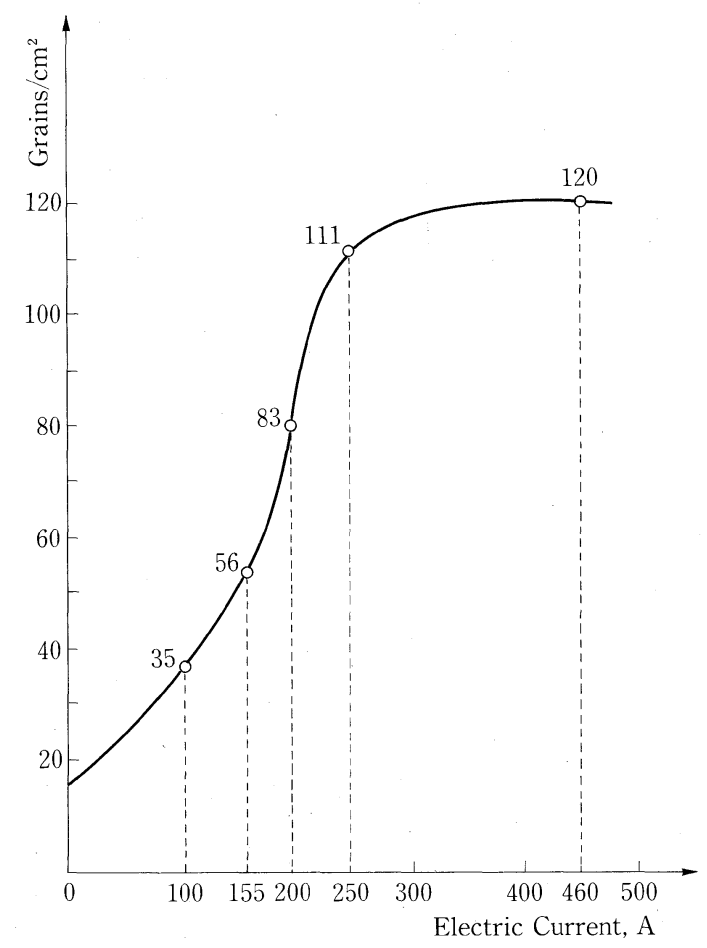

Fig. 9 The relation between the number of grains $/ \mathrm{cm}^{2}$ and the current. 


\section{Results and discussion}

\subsection{Casting with direct current}

The macrostructures of ingots subjected to varying direct current are shown in Fig (8). The chilling effect of the upper and lower electrodes is more pronounced in Fig (8a). The columnar grains are gradually replaced by equiaxed grains whose grain size becomes smaller and smaller. The number of grains $/ \mathrm{cm}^{2}$ was plotted versus the value of the current as shown in Fig (9). The slope is large at first and levels off after $250 \mathrm{~A}$ where the number of nucleating crystallites approaches to saturation.

\subsection{Casting in alternating magnetic field}

The macrostructures of small ingots cast in the absence and in the presence of varying magnetic field intensities are shown in Fig (10). It is evident that the structure was totally equiaxed grains only when the magentic field intensity was about 0.3 kilo-gauss. However, there appears a skin monolayer of bigger grains. The presence of these grains will be understood when the solidification process is elaborated. Alternating electromagnetic field creates induced emf. The resulting current interacts with the magnetic field to provide a force that causes stirring of molten metal. Stirring transports hot metal from the central region of the melt, which may melt back the solidification front. Thus, the molten metal instead of showing a marked temperature gradient toward the centre, has a comparatively uniform temperature. The temperature of the melt progressively decreases until the solidification point is reached, when the whole of the molten zone then solidifies almost at once. The motion at the same time creates crystallites from the solidification front that are transported into the hot molten metal. If there is a considerable degree of superheating, the crystallites

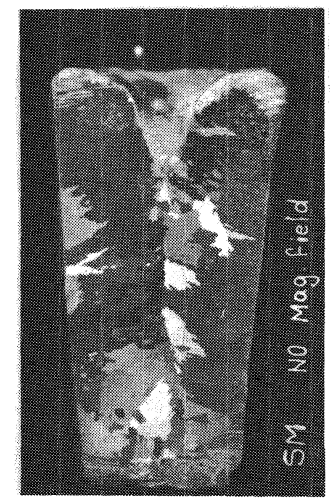

(a)

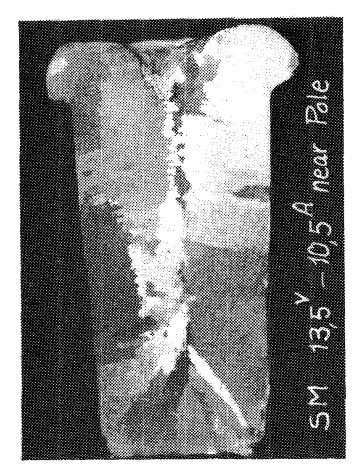

(d)

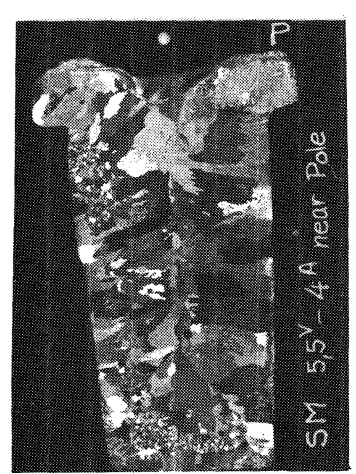

(b)

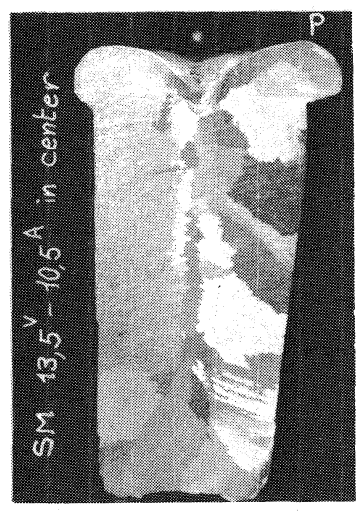

(e)

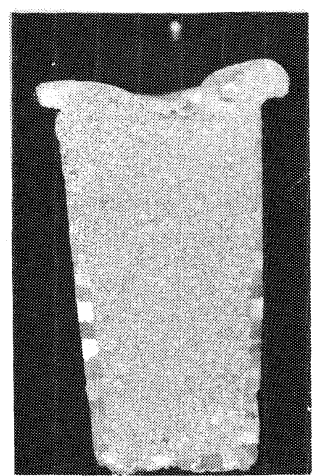

(c)

Fig. 10 Macrostructures of small ingots cast in alternating magnetic field at the following values of $H$ : a) zero, b) 0.32, c) 0.27 ,d) 0.73 ,e) 0.58 k-gauss. Magnification $=1$ 


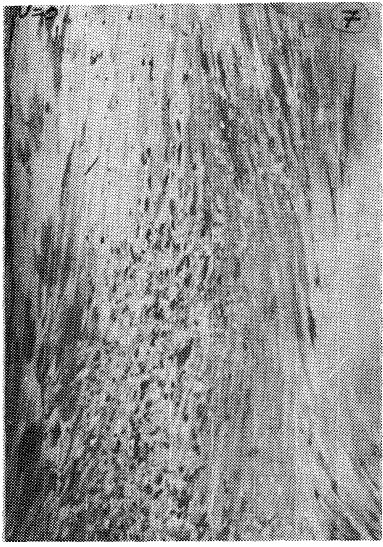

(a)

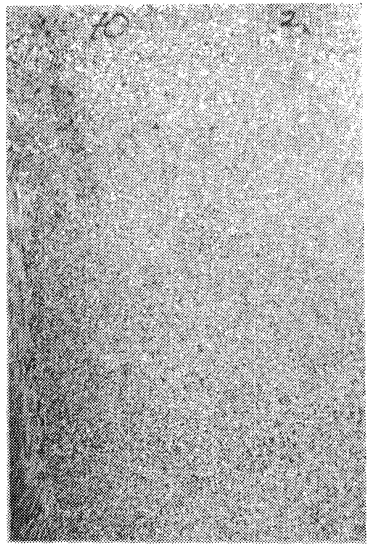

(b)

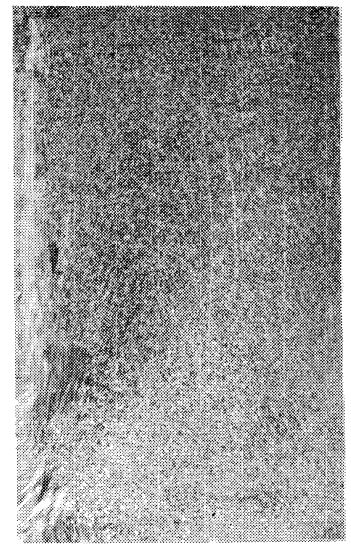

(c)

Fig. 11 Macrostructures of large ingots at the following values of $H$ : a) zero, b) 0.29 , c) 0.265 k-gauss. Magnification $=0.5$

remelt. As the temperature of molten metal becomes uniformly lower, the crystallites persist. As the solidification temperature is approached, the molten metal contains a high density of crystallites that nucleate and refine the grain size, giving rise to fine equiaxed structure. Number of columnar grains at the surface is decreased due to detachment as a result of the stirring effect. Moreover, the few grains left stay at a temperature near melting until the solidfication is completed and thus grow. Such grains are absent in the larger ingots because of the chilling effect of the cooling water.

The number of detached crystallites is expected to increase with increase of alternating magnetic field strength. However, as the magnetic field strength increases the undercooling decreases. This, in turn, increases the critical size of the nuclei. Consequently, the remelting of subcritical nuclei increases. The number of surviving nuclei seems to be the highest at $0.3 \mathrm{k}$-gauss. This explains the coarsening taking place at higher magnetic field strength. Tha macrostructures of large ingots in the absence and in the presence of varying magnetic field intensities are whown in Fig (11). The structure is totally equiaxed in Fig (11b) except at a small area outside the homogeneous magnetic field. The optimum value of the magnetic field intensity was about $0.3 \mathrm{k}$-gauss. The finest grain size in both cases yielded about 700 grains $/ \mathrm{cm}^{2}$ as determined by comparing with ASTM standards.

\section{Conclusions}

Applying direct current leads eventually to equiaxed grains of decreasing grain size as the current increases. However, the refining is not as efficient as in case of alternating magnetic field. In addition, the chilling effect of both electrodes and the resistance heating cannot be completely avoided. Casting in the presence of alternating magnetic field is easier and requires less precautions. There is an optimum value for the magnetic field at which the aluminium casting totally acquires fine equiaxed structure. This optimum value seems to be independent of the ingot size.

\section{Acknowledgements}

The authors wish to express their gratitude to all staff members and technicians of Material Technology Dept. of MTC. Thanks are also due to Dr. Ferenc Szabo of ALLUTERVFKI for designing and building the electromagnet.

\section{References}

1) R. Rumar: British Foundryman, 65 (1972), p. 56.

2) R. J. Southin: J. Inst. Met., 94 (1966), p. 401.

3) T. A. El-Bassyouni, A. A. Nofal, A. S. El-Sabbagh and M. K. Hussein: Egypt. J. Chem., 16, No 2, (1973), 
p. 143-52.

4) D. H. Lane, J. W. Cannighan and W. A. Tiller: Trans. Met. Soc. AIME, 218 (1960), p. 985.

5) W. C. Johnson, G. R. Kotler, S. O'Hara, H. V. Aschom and W. A. Tiller: Trans. Met. Soc. AIME, 233 (1965), p. 1956.

6) D. R. Milner: Dept. of Ind. Met., Univ. of Birmingham, England, Paper presented at a seminar ASM, Oct. 1974.

7) Z. N. Getselev, J. of Metals, Oct. (1971), p. 38.

8) S. Asai, K. Yasui and I. Muchi, Trans. ISIJ, 18 (1978), p. 754.

9) D. R. Uhlmann, T. P. Seward, and B. Chalmers: Trans. Met. Soc. AIME, 236 (1966), p. 527.

10) T. Umeda: Report of the 11th Meeting of Solidification Committee, Joint Soc. on Iron and Steel Basic Research, ISIJ, Rep. No 11-II-3, 1975.

11) T. Momono, M. Tamada and K. Ikawa: J. Jap. Foundrymen's Soc., 47, 1975, p. 477.

12) M. D. Sahu and V. A. Gadgil: The British Foundryman, 70 (1977), p. 89. 